\title{
Mechanistic understanding of 3D printed polycarbonate process yielding comparable dielectric strength with injection molding process
}

\author{
Anshita Sudarshan ${ }^{1}$ (i) . S. M. Swamy ${ }^{1} \cdot$ Nitesh Shet $^{1} \cdot$ Hari Prasad $^{1} \cdot$ Juha-Matti Levasalmi ${ }^{2}$. \\ Prasanta Mukhopadhyay ${ }^{1}$
}

Received: 17 October 2019 / Accepted: 12 May 2020 / Published online: 5 June 2020

(c) Springer Nature Switzerland AG 2020

\begin{abstract}
Plastic components used as insulators for electrical segments are conventionally processed by injection molding (IM). One of the important considerations in electrical safety is dielectric strength. There is a growing interest to process such components using additive manufacturing (3D printing) due to the ease of fabricating complex component designs without elaborate machinery while retaining similar property attributes. This study established a mechanistic understanding of dielectric breakdown in 3D (fused filament fabrication) printed polycarbonate. The impact of 3D printing process parameters on the dielectric performance is established. Appearance of dielectric breakdown site on the sample was found to be a good indicator of its dielectric performance. The optical imaging studies revealed that IM sample which exhibited a dielectric strength of $23.4 \mathrm{kV} / \mathrm{mm}$ (ASTM D149) was associated with a pin-centric, radially symmetric and cohesive crack measuring $450 \mu \mathrm{m}$ at the site of dielectric breakdown. It was found that 3D printed samples exhibiting inferior dielectric strength (7.2-19.9 kV/mm) were associated with larger, elongated, radially asymmetric and non-cohesive crack lengths. An inverse correlation between dielectric strength and length of the dielectric breakdown (cracks) is elucidated in this study. The optimized print parameters resulted in a breakdown that appeared to be cohesive, radially symmetric and which ultimately correlated with an improved dielectric strength $(22.4-23.2 \mathrm{kV} / \mathrm{mm})$, at par with IM.
\end{abstract}

Keywords 3D printing · Additive manufacturing · Fused filament fabrication · FFF - Dielectric strength - Optical microscopy

\section{Introduction}

Rapid prototyping is used in a variety of industries to fabricate parts relatively quickly before product commercialization. The intent here is to create prototypes in quick time, further to which part consolidation is possible. Incidentally, the application of additive manufacturing (also referred to as 3D printing) in rapid prototyping started being adopted by the industry way back in late 1980s [1-3]. However, the industry has matured a long way since then. Significant improvements in 3D printing machines have meant they are not merely used for prototype designs, but directly for final parts and components [3]. Unlike conventional manufacturing (e.g., injection molding), 3D printing brings along the ease of fabricating complex designs without elaborate machinery. The

SABIC and brands marked with ${ }^{\mathrm{TM}}$ are trademarks of SABIC or its subsidiaries or affiliates, unless otherwise noted. Any brands, products or services of other companies referenced in this document are the trademarks, service marks and/or trade names of their respective holders.

Anshita Sudarshan, anshita.sudarshan@sabic.com | 'SABIC Research and Technology Pvt. Ltd., Sarjapura - Attibele Road, Bengaluru, Karnataka 562125, India. ${ }^{2}$ SABIC, 1 Noryl Avenue, Selkirk, NY 12158, USA. 
model for an additive manufacturing technology is generated using a three-dimensional computer-aided design (3D CAD) system. Hereafter, the process involves fabricating parts made by adding materials in layers, where each layer is a thin cross section of the part derived from the original CAD data. While healthcare applications were the first to benefit, recent advances in 3D print technology have seen interest in making functional parts for industries such as mass transportation, automotive, aerospace and electronics. For such newer applications, in addition to mechanical, thermal and flow, electrical properties will assume significance.

At present, there are many known additive manufacturing processes: selective laser sintering, stereolithography, laminated object manufacturing, inkjet printing, polymer jetting, fused filament fabrication to mention a few [4-7]. Each of these technologies brings in its own set of advantages as well as limitations depending upon the various process parameters involved [8]. Currently, fused filament fabrication (FFF) has the widest choice of commercially available engineering thermoplastic that can meet different functional properties. The various print parameters involved in the construction of design are build plane, bead width, layer thickness, build angle, print speed $(\mathrm{mm} / \mathrm{s})$ and air gaps between the adjacent tracks [9-12]. All these process parameters decide the quality as well as properties of the final 3D printed part produced from a fused filament fabrication machine.

FFF fabricates designed parts from polymeric resins by stacking layers of semi-molten filament upon a build platform. In such a process, the liquefier header deposits one layer of material in a slice upon a build platform, by moving across axes. The material filament is pulled from the material pool through drive wheels. It is then heated inside the liquefier head which is extruded through a nozzle to build successive layers which fuse together to give the desired 3D part. The schematic of FFF process is shown in Fig. 1. For FFF process, many research papers have studied structure-property correlation where printing parameters influenced the dielectric performance [8, 13-17].

The motivation around this work was, therefore, to generate insights around key 3D printing process parameters and their effect on electrical properties, specifically dielectric strength. The buildup of electric field stress on the insulated polymeric part causes part breakdown leading to loss of material integrity. A recent study by Hoff et al. has carried out a study of dielectric behavior of ABS vis-avis different additive manufacturing techniques $[18,19]$. Our current paper focusses on creating in-depth understanding on dielectric breakdown mechanism in different orientations created by FFF printing technology. There is ample literature on polymeric dielectric breakdown mechanisms by IM $[8,9,17,20]$. However, there is insufficient

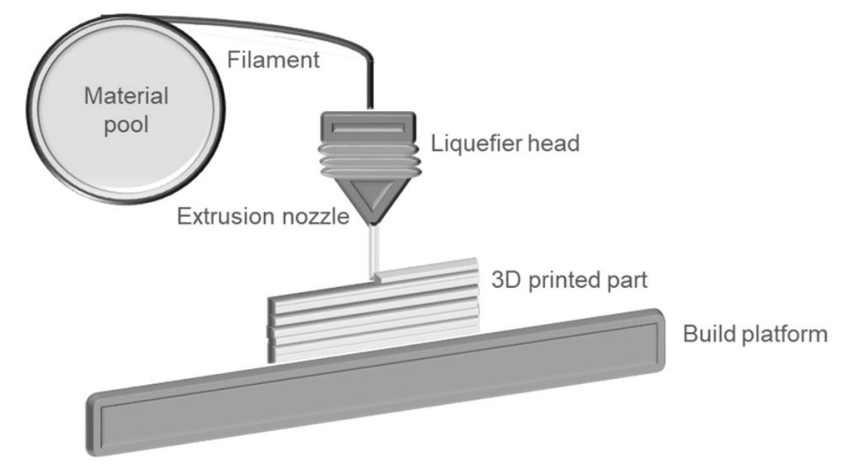

Fig. 1 Schematic of fused filament fabrication process

literature reporting the dielectric breakdown mechanism in 3D printed parts. In this work, the impact of 3D printing parameters on the dielectric performance has been studied. This understanding aided to optimize printing parameters such that 3D printed parts exhibited comparable dielectric strength to IM.

\section{Materials and methods}

\subsection{Materials}

Sample specimens for dielectric studies were made using $\mathrm{IM}$ as well as $3 \mathrm{D}$ printing (fused filament fabrication process). The test specimens used were from the same manufacturing batch to rule out any batch-to-batch variation. Sample plaques were made of size $60 \mathrm{~mm}$ (length) * $60 \mathrm{~mm}$ (width) * $1.5 \mathrm{~mm}$ (thickness), using polycarbonate (LEXAN ${ }^{\mathrm{TM}}$ AMHF1110F filament) manufactured by SABIC.

\subsection{Methodology}

Figure 2 is an algorithm presented that provides understanding on the methodology pursued in this study. It provides an overview on the material started with, how these are processed and finally which properties are of interest to study.

\subsubsection{Samples prepared from injection molding process}

A $130 \mathrm{~T}$ Injection molding machine (Sumitomo ${ }^{\circ}$ ) was used to prepare injection-molded specimens. A schematic of sample preparation using an injection molding process is shown in Fig. 3. Polycarbonate material was injection-molded at melt temperature $\left(285^{\circ} \mathrm{C}\right)$, mold temperature $\left(80^{\circ} \mathrm{C}\right)$, injection pressure $(860 \mathrm{bar})$, injection speed $(20 \mathrm{~mm} / \mathrm{s})$, injection time $(0.85 \mathrm{~s})$, holding pressure (780 bar), holding time ( $9 \mathrm{~s})$, cooling time (15 s) and 


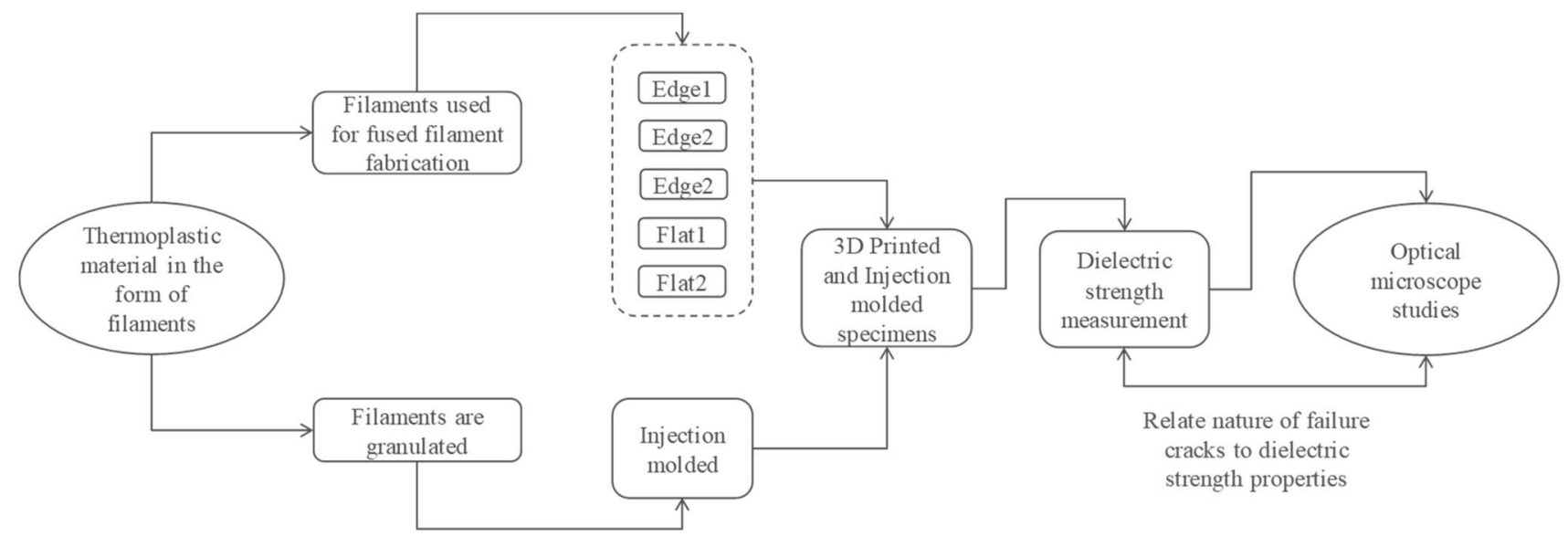

Fig. 2 Algorithm on the process and the testing methodology

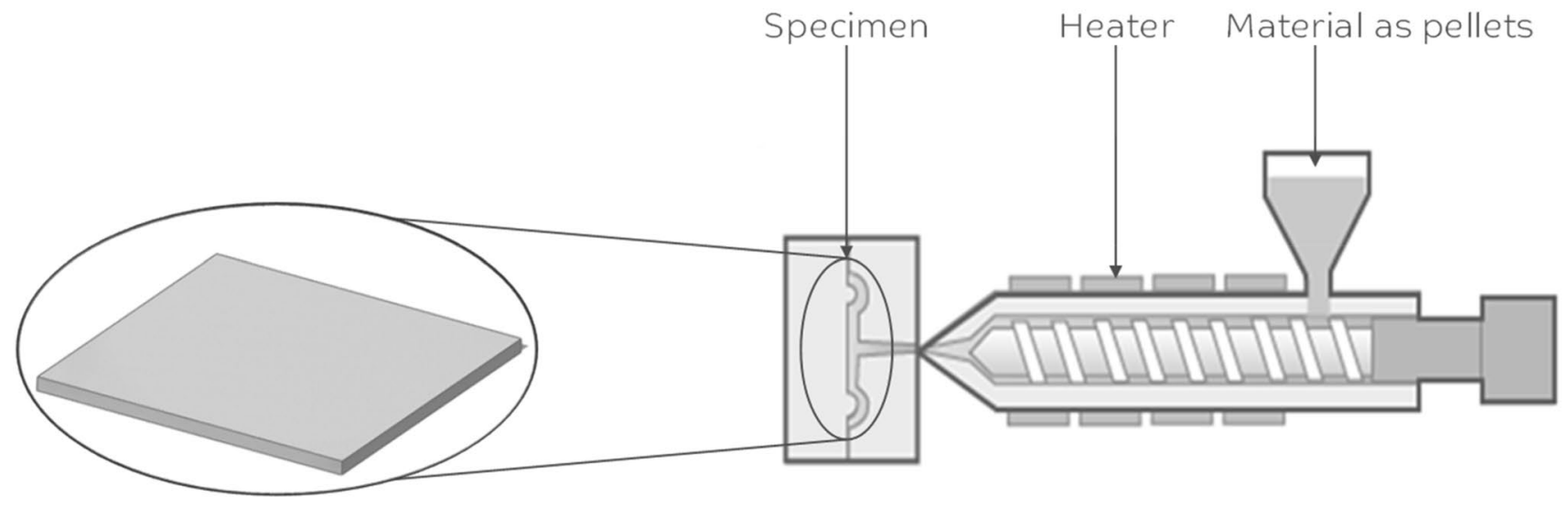

Fig. 3 Schematic of sample preparation using an injection molding process

cycle time (32 s). All the samples were pre-conditioned at $23 \pm 2{ }^{\circ} \mathrm{C}$ and $50 \pm 10 \%$ relative humidity for a minimum of $40 \mathrm{~h}$ (ASTM D618) to allow the samples to attain equilibrium with room conditions.

\subsubsection{Samples prepared from fused filament fabrication process}

Fortus 450 (Stratasys ${ }^{\oplus}$ ) printer was used to prepare 3D printed specimens, to evaluate dielectric breakdown using the fused filament fabrication process. A schematic of the sample preparation using fused filament fabrication process is demonstrated in Fig. 4.

The bead width was varied from 0.4064 to $0.7314 \mathrm{~mm}$, commensurate with the T-16 tip from Stratasys ${ }^{\circledR}$ Fortus ${ }^{\circledR} 450$ machine. Compactness in the specimens was altered by varying primary print parameters such as, build plane, build angle and bead/contour width (Fig. 5a). Parameters including layer thickness/height, number of contours and part interior shape were kept constant (Fig. 5b). The layer height (also known as slice height) considered for our experiment was kept constant, $0.254 \mathrm{~mm}$.

Based on the FFF print parameters (Fig. 5a), build planes, build angle and bead width were varied and these samples are designated in this study as Edge1, Edge2, Edge3, Flat1 and Flat2.

\subsubsection{Dielectric strength measurement}

Dielectric strength is one of the key properties to evaluate its electrical insulation resistance typically materials like plastics. Depending on the type of application, the dielectric strength can be measured using AC or DC voltages. In this study, we have used $A C$ voltage using highvoltage $A C$ dielectric test equipment (HIPOTRONICS ${ }^{\circ}$ ). The spread of electrical charges around electrode is restricted by oil when high voltage is applied. Thus, to obtain consistent results, the test was carried out in an oil medium. Samples were conditioned for a minimum of $48 \mathrm{~h}$ in a room maintained at $23 \pm 2{ }^{\circ} \mathrm{C}$ with $50 \pm 10 \%$ 


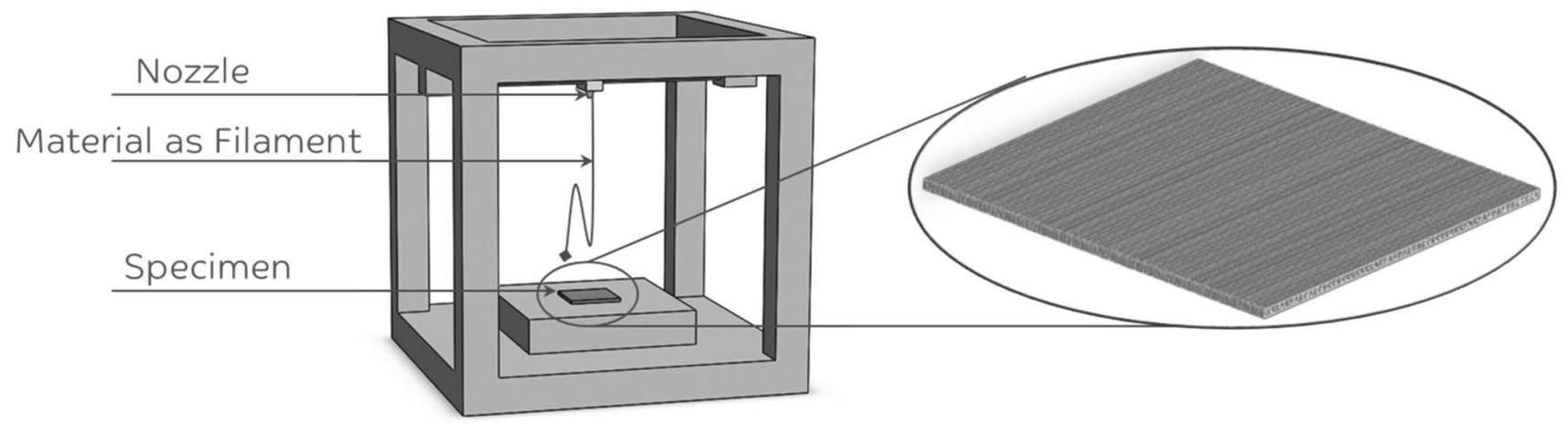

Fig. 4 Schematic of sample preparation using fused filament fabrication process

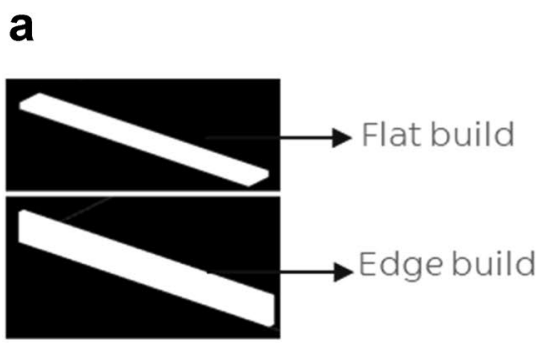

Build plane

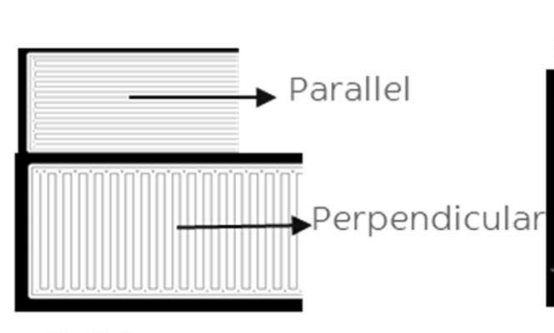

Build angle

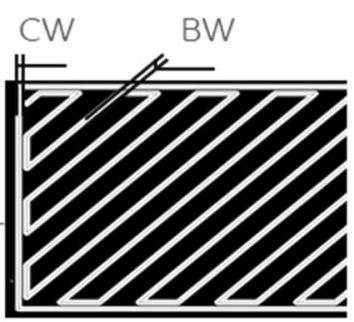

Bead width
Bead width $(B W)=$ Contour width (CW)

\section{b}

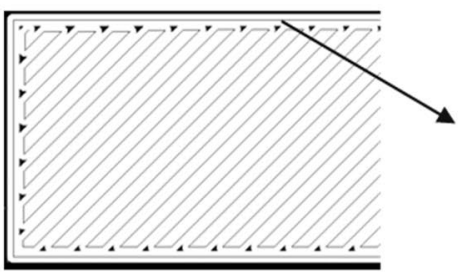

Contour

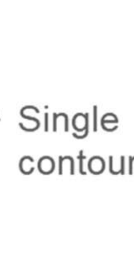

Single

(1)

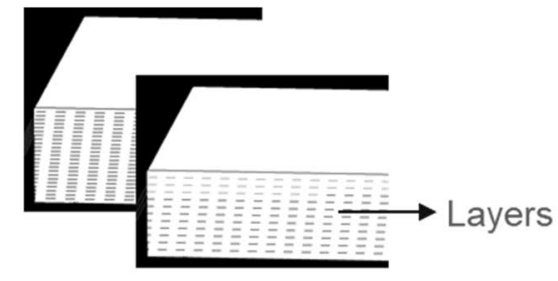

Layer height

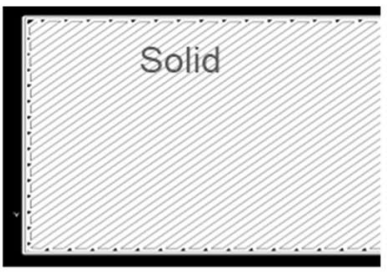

Part inside

Fig. 5 a Pictorial representation of varied parameters (build plane, build angle, bead width) in machine settings and $\mathbf{b}$ constant parameters (contour, layer height, in-side part) in machine settings

relative humidity (ASTM D618). Pre-conditioned specimens were kept in between the flat surfaces of the electrodes; voltage was raised until breakdown occurred in the specimens. Electric voltage was applied across the sample with the help of two electrodes (ASTM D149 Electrode type-2). For better comparative studies, short time method (Method A, ASTM D149) was adopted. The samples were subjected to a constant rate of voltage rise of $500 \mathrm{~V} / \mathrm{s}$ across the specimen until the failure occurred. Peak breakdown voltage was noted. The samples were cleaned thereafter to remove oil stains. Thickness of the samples was measured near the cavity area, and the dielectric strength was calculated by dividing the breakdown voltage by the thickness of the specimen. The calculation is as given below:

Di-electric strength $(\mathrm{kV} / \mathrm{mm})$

= Breakdown voltage $(\mathrm{kV}) /$

Thickness of the specimen $(\mathrm{mm})$.

\subsubsection{Optical imaging}

KEYENCE ${ }^{\circ}$ VHX-5000 optical microscope was equipped with a 54 megapixel CCD detector with lens

\section{SN Applied Sciences}


magnification up to $5000 \times$. 3D images were captured using the ability of the in-built software to vary the focal plane along the $z$-direction. Optical images in both 2D and 3D were recorded.

\section{Results and discussion}

Dielectric breakdown in polymers is the event of electrical insulation failure by virtue of its inability to withstand the applied electric field. Ostensibly, the adverse effects of anisotropy on dielectric performance of 3D printed polymers are known $[18,19]$. However, it is imperative to garner holistic understanding of this structure-property relationship. Figure 6 showed a plot of the dielectric strength of polycarbonate specimen prepared from injection molding and 3D printing. An average dielectric strength of $23.2 \mathrm{kV} /$ $\mathrm{mm}$ was observed for polycarbonate injection-molded specimens. However, for 3D printed specimen dielectric strength varied from 7.2 to $23.4 \mathrm{kV} / \mathrm{mm}$ across different print parameters. Figure 6 also indicated variability in dielectric strength within a 3D printed sample.

The dielectric strength in Edge1 and Flat1 was inferior, while that in Edge2, Flat2 and Edge3 was equivalent to injection-molded specimens. Optical microscopy enabled to discern the root cause of variability in dielectric performance of 3D printed samples by examining the site of dielectric strength failure. Optical 3D micrographs at $200 \times$ magnification (Fig. 7a-f) helped in determining the differences in dielectric failure pathways across the thickness of the sample, while 2D micrographs at $200 \times$ magnification (Fig. $7 \mathrm{~g}-\mathrm{I}$ ) revealed the size and shape of the cavity on the surface. Figure $7 \mathrm{a}, \mathrm{g}$ reveals that injection-molded polycarbonate sample formed a very circular, cohesive and "pin-centric" crack. The diameter of the crack was around $450 \mu \mathrm{m}$. Edge1 (Fig. 7f, I) and Flat1 (Fig. 7d, j) exhibited inferior dielectric strength than IM. Edge1 and Flat1 did not show a "pin-centric" crack. The site of dielectric breakdown in these build orientations showed a corresponding deterioration in terms of charred, larger, elongated, non-cohesive cracks measuring $525 \mu \mathrm{m}$ and $750 \mu \mathrm{m}$, respectively. The dielectric strength was observed to be drastically low for Flat $1(7.2 \mathrm{kV} / \mathrm{mm})$ and deteriorated for Edge1 (19.9 kV/ $\mathrm{mm})$. This immoderate decline in Flat 1 was evident in the 2D and 3D images (Fig. 7d, j), where the cavity created by dielectric breakdown was the most irregular shaped. Multiple breakdown pathways were observed in these samples as visualized through 3D micrographs. A qualitative understanding of the cracks related to properties has been reported earlier, where the authors attributed the inferior properties to air voids [18].

3D optical micrographs illustrated the progression of the crack across the material. Figure 8 a represents a material failure in a typical injection-molded sample signifying a pin-centric, radially symmetric and cohesive pathway. An injection-molded sample having homogenous material density imparts consistent resistance to the applied electric field. The homogeneity resulted in a breakdown puncture due to concentric leakage pathway for high voltage, whereas Fig. $8 \mathrm{~b}$ represents material failure in a fused filament fabricated specimen with inferior dielectric strength. It demonstrated multiple leakage pathways due to material incongruence and inhomogeneity. The fabrication of polymer during FFF creates trough and crest structure due to bead-by-bead deposition of the molten polymer. This process may cause inhomogeneity in material density in the polymer sample. The printing processing conditions creates a difference in material deposition. It
Fig. 6 Graphical representation showing dielectric strength of polycarbonate specimens prepared from IM and $3 \mathrm{D}$ printing

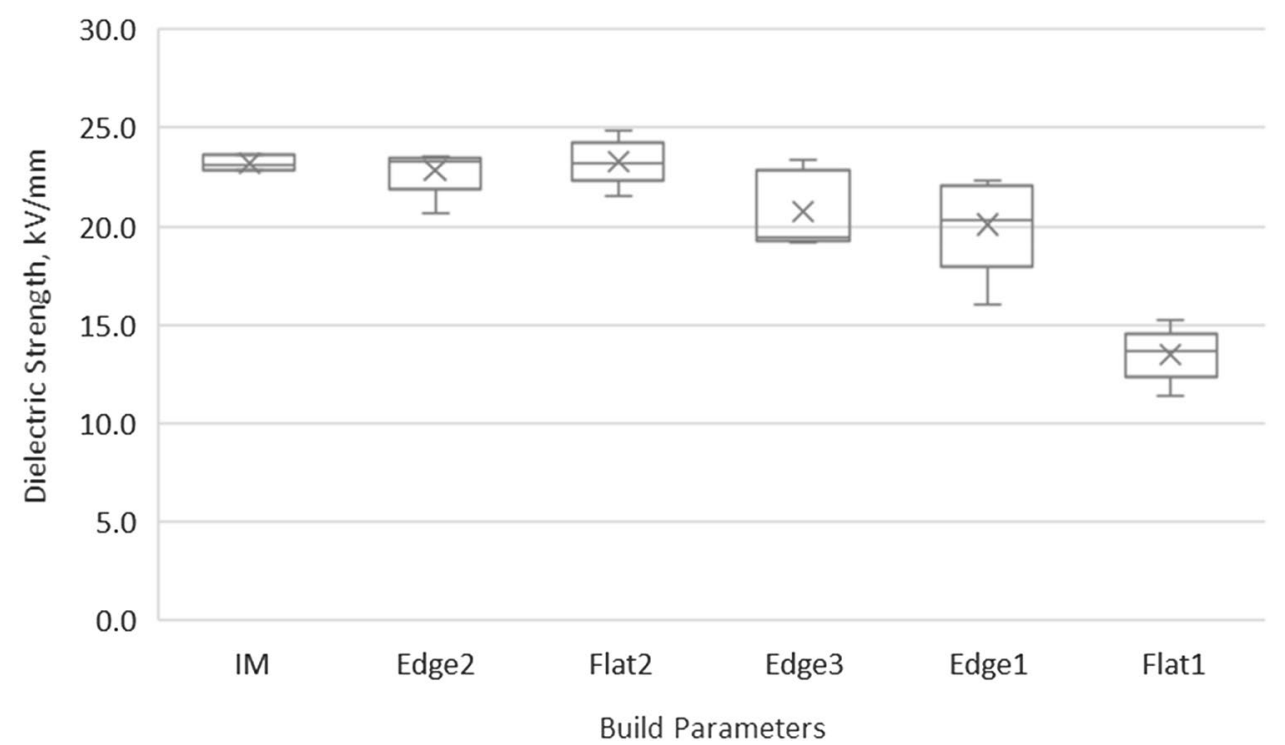

SN Applied Sciences 


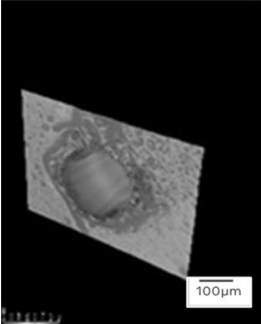

(a)

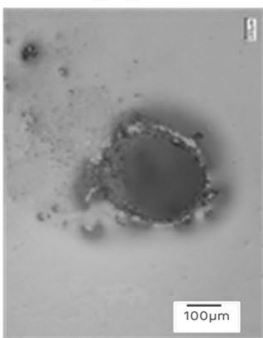

(g)

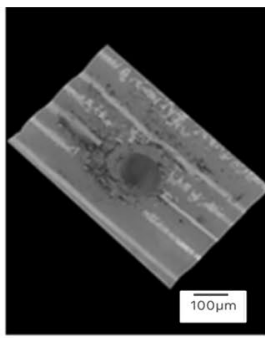

(b)

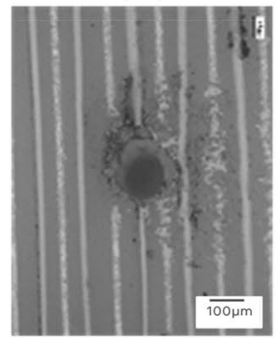

(h)

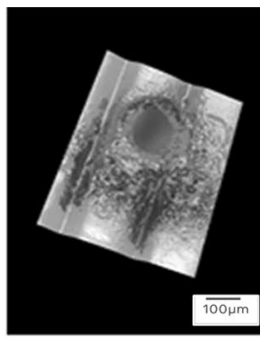

(c)

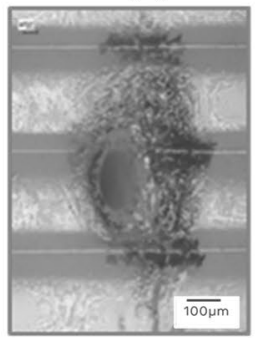

(i)

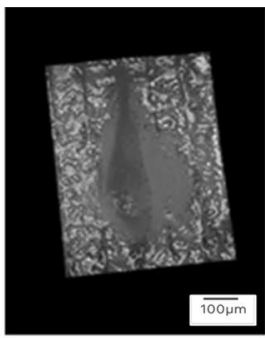

(d)

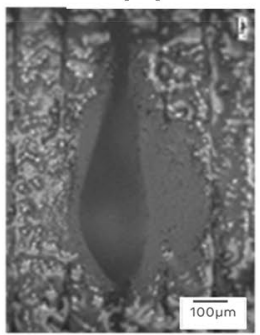

(j)

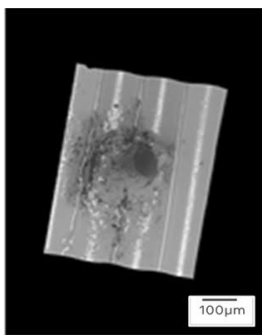

(e)

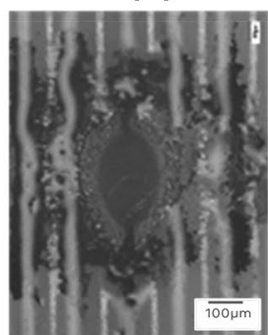

(k)

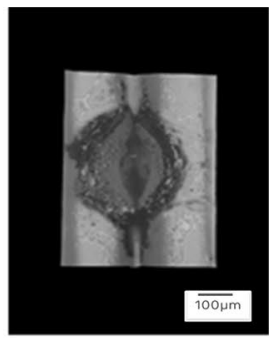

(f)

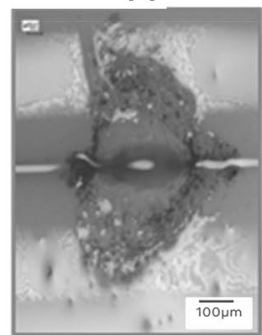

(I)

Fig. 7 Post-failure 3D (a-f) and 2D (g-t) optical micrographs ( $200 \times$ magnification) of PC specimen $\mathbf{a}, \mathbf{g}$ injection-molded; $\mathbf{b}, \mathbf{h}$ Edge2; $\mathbf{c}, \mathbf{i}$ Flat2; d, j Flat1; e, k Edge3; f, I Edge1

Fig. 8 Three-dimensional cross section view representing (a) concentric leakage pathway and $\mathbf{b}$ multiple leakage pathway

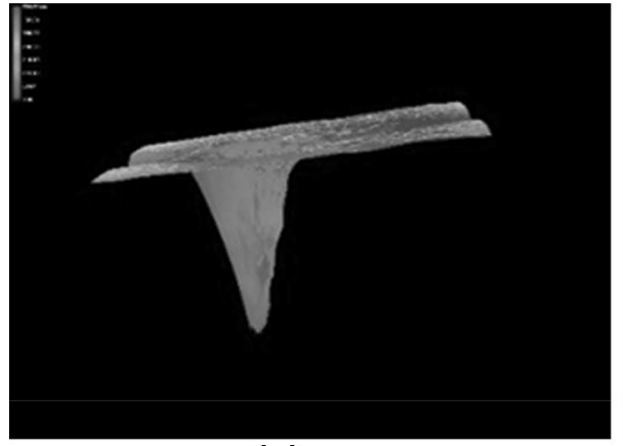

(a)

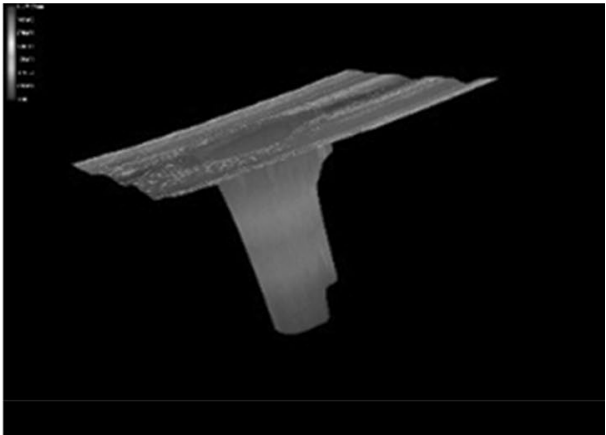

(b) is through these channels of material density inhomogeneity imparting the sample anisotropy across the sample, which may lead to multiple leakage pathways during the course of dielectric breakdown. Based on this understanding that presence of multiple leakage pathways for high voltage during dielectric breakdown causes a deterioration in the performance of 3D printed samples, the print parameters were further optimized with respect to build angle, build orientation and bead width. This resulted in samples Edge2, Edge 3 and Flat 2 with improved dielectric strength performance.

Edge2, Flat2 and Edge 3 exhibited dielectric strength performance of $23.4 \mathrm{kV} / \mathrm{mm}, 23.1 \mathrm{kV} / \mathrm{mm}$ and $22.4 \mathrm{kV} /$ $\mathrm{mm}$. The cracks appeared cohesive. Corresponding crack lengths measured were $250 \mu \mathrm{m}, 270 \mu \mathrm{m}$ and $325 \mu \mathrm{m}$ for Edge2, Flat2 and Edge3, respectively. Cohesive nature of crack was indicative of radially symmetric breakdown denoting the absence of multiple leakage pathways. The breakdown appearance was similar to the cracks in the injection-molded sample. Edge2, Flat2 and Edge3 were characterized by pin-centric and cohesive breakdown pathway in 3D micrographs as shown in Fig. 7b, h, c, i and $\mathrm{e}, \mathrm{k}$, respectively. It was now evident that in order to achieve comparable dielectric strength to that of $I M$, the appearance of the dielectric breakdown should be coherent and have a radially symmetric breakdown pathway.

From Fig. 9, it is apparent that 3D printed samples of polycarbonate demonstrated an inverse relationship between the crack length and the dielectric strength. As the crack length became progressively larger due to neighboring routes of leakage pathways at the site of dielectric failure, the dielectric strength became further inferior. As seen in 2D and 3D micrographs of Fig. 7, the crack length elongation occurs along the axis of build orientation.

\section{SN Applied Sciences}




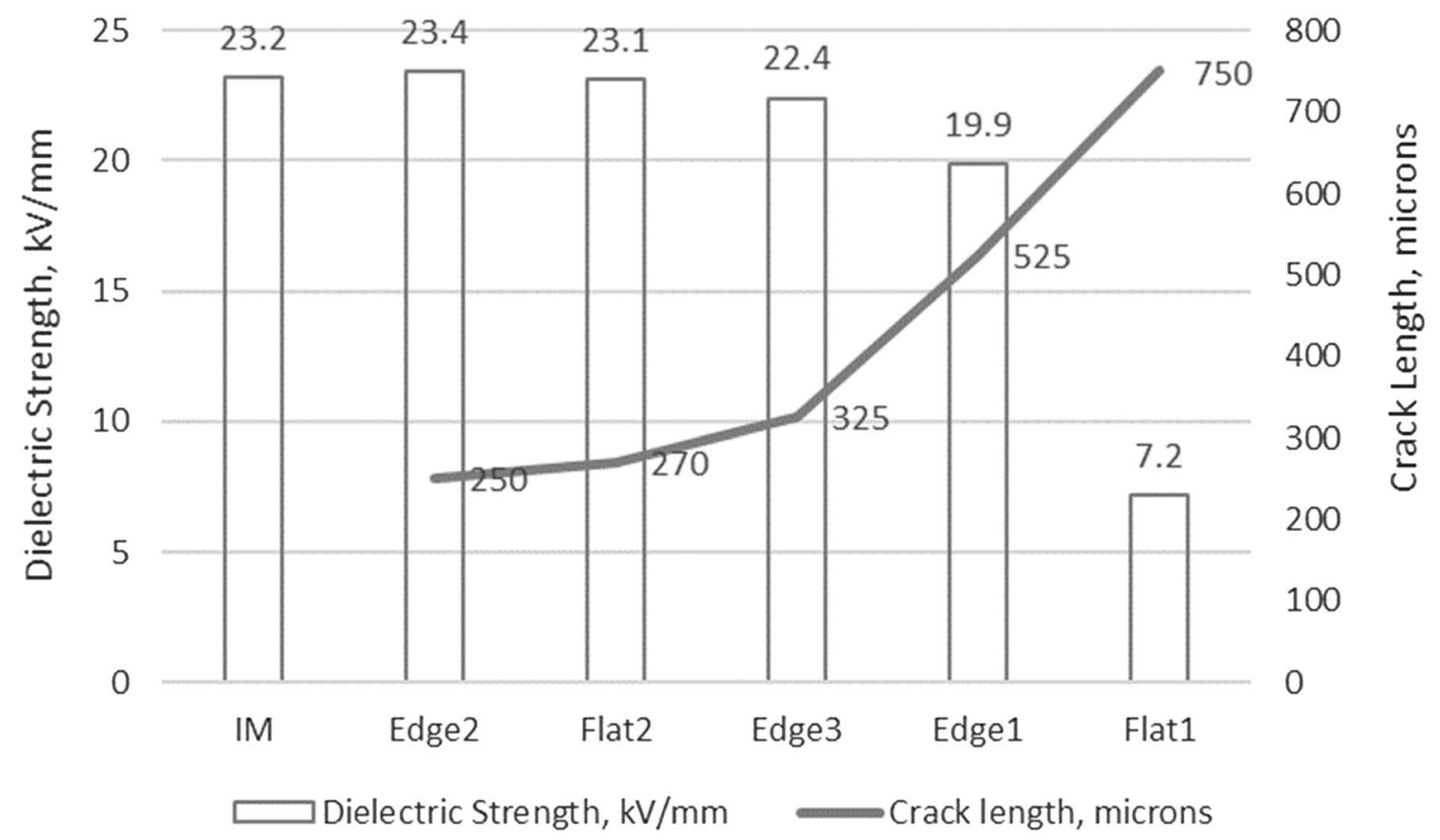

Fig. 9 Graphical representation showing correlation of dielectric breakdown with crack length in polycarbonate

Edge2, Flat2 and Edge3 oriented polycarbonate samples withstood applied voltage homogenously and hence performed equivalent to injection-molded sample. On the other hand, Edge1 and Flat1 polycarbonate samples implied material inhomogeneity inhibiting its isotropic ability to withstand the electric field. This emphasized the influence of bead width, orientation plane or build angle, which may create leakage pathways within the printed material. Hence, this work highlights the need for optimization of process parameters to enhance polymer performance. Experiments with acrylonitrile-butadiene-styrene and polyetherimide (by the authors) also displayed consistent mechanisms and correlation of crack length with dielectric performance (results not shared in the present publication). Further work can be undertaken to understand other contributing factors for dielectric strength in $3 \mathrm{D}$ printed samples covering other engineering thermoplastics resins.

\section{Conclusions}

Additive manufacturing offers multiple benefits, such as opportunity for mass customization, part consolidation and design flexibility. A critical need in the industry is to address mass manufacturability of such a process. The purpose of this work was to establish structure-property relationship between the 3D printed polycarbonate using FFF process and dielectric strength. The optical imaging of IM and 3D printed polycarbonate samples revealed differences in the cavity formed during dielectric breakdown. The study demonstrated inhomogeneity in polymer deposition during FFF process, which influenced the dielectric strength performance deleteriously. The length of cavity formed during the dielectric breakdown correlates negatively with the dielectric strength performance. A larger crack length was suggestive of more inferior dielectric property. In addition, the crack appearance correlated qualitatively to the dielectric strength performance. Non-cohesive, radially asymmetric, charred and elongated cavities during dielectric breakdown were associated with inferior dielectric strength performance. The material inhomogeneity in FFF printed parts facilitated multiple leakage pathways during dielectric failure leading to inferior performance. These leakage pathways were typically in the direction of build orientation signifying the anisotropy associated with FFF printing. The print parameters such as bead width, build angle or build orientation were varied to assess the influence on the material property. The 3D optical imaging provided insights into the failure pathways across the sample thickness. Based on this understanding, printing parameters were optimized to achieve dielectric strength commensurate with $\mathrm{IM}$. The optimized 3D printed samples had a dielectric breakdown with cavities similar to IM, consistent with their comparable dielectric strength. The appearance of dielectric breakdown in these samples was found to be 
a pin-centric, cohesive and well-defined circular shaped cavity. This study enables better understanding during product development and design optimization for mass manufacturing of 3D printed parts that require adequate insulation characteristics.

Acknowledgements We would like to express our sincere gratitude to Sumanda Bandyopadhyay and Vandita Pai-Paranjape for sharing several insights during the course of this research.

Author contributions SMS and HP contributed to dielectric strength measurement; AS helped in optical measurements and analysis; NS helped in 3D printing and designing; , PM, J-ML and AS contributed to conceiving and designing the experiments. All authors have contributed toward writing the paper and its related analysis and interpretations.

\section{Compliance with ethical standards}

Conflict of Interest On behalf of all authors, the corresponding author states that there is no conflict of interest.

\section{References}

1. Kruth JP (1991) Material incress manufacturing by rapid prototyping techniques. CIRP Ann. https://doi.org/10.1016/S0007 -8506(07)61136-6

2. Kruth JP (1995) New application of physical and chemical processes for material accretion manufacturing. In: Proceedings of the 11 th international symposium on electro-machining, pp 3-29

3. Kruth JP, Leu MC, Nakagawa T (1998) Progress in additive manufacturing and rapid prototyping. CIRP Ann Manuf Technol. https ://doi.org/10.1016/S0007-8506(07)63240-5

4. Singh S, Ramakrishna S, Singh R (2017) Material issues in additive manufacturing: a review. J Manuf Process. https://doi. org/10.1016/j.jmapro.2016.11.006

5. Gibson I, Rosen DW, Stucker B (2009) Additive manufacturing technologies: rapid prototyping to direct digital manufacturing. Springer, New York

6. Brandt M (2017) Laser additive manufacturing: materials, design, technologies and applications, 1st edn. Woodhead, Boston

7. Kotlinski J, Kesy A, Kesy Z, Jackson M, Parkin R (2009) Dimensional deviations of machine parts produced in the laser sintering process. Int J Rapid Manuf. https://doi.org/10.1504/IJRAP IDM.2009.028933
8. Lee CS, Kim SG, Kim HJ, Ahn SH (2007) Measurement of anisotropic compressive strength of rapid prototyping parts. J Mater Process Technol. https://doi.org/10.1016/j.jmatprotec .2006.11.095

9. Ahn SH, Montero M, Odell D, Roundy S, Wright PK (2002) Anisotropic material properties of fused deposition modeling ABS. Rapid Prototyp J. https://doi.org/10.1108/13552540210441166

10. Budzik G (2010) Geometric accuracy of aircraft engine blade models constructed by means of generative rapid prototyping methods FDM and SLA. Adv Manuf Sci Technol 34(1):33-43

11. Xu F, Loh HT, Wong YS (1999) Considerations and selection of optimal orientation for different rapid prototyping systems. Rapid Prototyp J. https://doi.org/10.1108/13552549910267344

12. Lee BH, Abdullah J, Khan ZA (2005) Optimization of rapid prototyping parameters for production of flexible ABS object. J Mater Process Technol. https://doi.org/10.1016/j.jmatprotec .2005.02.259

13. Comb JW, Priedeman WR, Turley PW (2002) Layered manufacturing control parameters and material selection criteria. J Manuf Sci Eng 68-2:547-556

14. Allen S, Dutta D (1998) Wall thickness control in layered manufacturing for surfaces with closed slices. Comput Geom Theory Appl 10(4):223-238

15. Alexander P, Dutta D (2000) Layered manufacturing of surfaces with open contours using localized wall thickening. Comput Aided Des. https://doi.org/10.1016/S0010-4485(99)00079-2

16. Han WB, Jafari MA, Seyed K (2007) Process speeding up via deposition planning in fused deposition-based layered manufacturing processes. Rapid Prototyp J. https://doi.org/10.1108/13552 540310489596

17. Sun Q, Rizvi GM, Bellehumeur CT, Gu P (2008) Effect of processing conditions on the bonding quality of FDM polymer filaments. Rapid Prototyp J. https://doi.org/10.1108/1352554081 0862028

18. Hoff BW, Maestas SS, Hayden S, Harrigan DJ, Grudt RA, Ostraat ML, Horwath JC, Leontsev S (2018) Data on dielectric strength heterogeneity associated with printing orientation in additively manufactured polymer materials. Addit Manuf. https://doi. org/10.1016/j.addma.2018.04.010

19. Monzel WJ, Hoff BW, Maestas SS, French DM, Hayden SC (2015) Dielectric breakdown of additively manufactured polymeric materials. IEEE Trans Dielectr Electr Insul. https://doi. org/10.1109/TDEl.2015.005199

20. leda M (1980) Dielectric breakdown process of polymers. IEEE Trans Electr Insul. https://doi.org/10.1109/TEI.1980.298314

Publisher's Note Springer Nature remains neutral with regard to jurisdictional claims in published maps and institutional affiliations. 